\title{
Phage lysin to control the overgrowth of normal flora in processed sputum samples for the rapid and sensitive detection of Mycobacterium tuberculosis by luciferase reporter phage assay
}

\author{
Balaji Subramanyam, Gomathi Sivaramakrishnan, Azger Dusthackeer and Vanaja Kumar*
}

\begin{abstract}
Background: Phage lysin, extracted from three bacteriophages was used in place of antibiotics to control the overgrowth of normal flora in processed sputum samples leading to the sensitive detection of Mycobacterium tuberculosis using diagnostic luciferase reporter phage assay (DLRPA).
\end{abstract}

Methods: A total of 129 sputum samples were processed by modified Petroff's method. Two Lowenstein Jensen slopes were inoculated from the processed sputum deposit thus obtained. The remaining deposits were transferred to $7 \mathrm{ml}$ of Middlebrook $7 \mathrm{H} 9$ complete medium supplemented with phage lysin and incubated at $37^{\circ} \mathrm{C}$. DLRPA was done using phAE129 at days 7, 9,14 and 21. At the end of day 21, the samples were centrifuged and the pellets were inoculated on to 2 more $L J$ slopes to validate DLRPA results.

Results: The sensitivity and specificity of DLRPA in detecting M. tuberculosis from sputum specimens was $90 \%$ and $81 \%$ respectively compared to conventional $L$ culture. The agreement between the methods was $87 \%$. The rate of contamination for DLRPA using phage lysin was 9.3\%.

Conclusion: Phage lysin can be used to decontaminate sputum samples for the detection of M. tuberculosis by DLRPA directly from processed sputum specimens.

Keywords: DLRPA, M. tuberculosis, Rapid Diagnosis, Phage lysin

\section{Background}

Lysin, en enzyme produced by bacteriophages, lyses the bacterial cell wall of active host cells to release progeny phages. Though phage lysins are produced inside host bacteria and normally cause lysis from within, they also have external strong lytic activity towards closely related bacteria [1]. Lysins are potential antibacterials regardless of the antibiotic sensitivity of host cells and they exhibit low probability of developing resistance [2]. Phage lysins have been used extensively as an alternative to antibiotics for the targeted killing of bacterial pathogens $[3,4]$.

Phagebiotics consisting of a cocktail of three bacteriophages were used to substitute for antibiotics in liquid media to control the overgrowth of normal flora in

\footnotetext{
* Correspondence: vanaja_kumar51@yahoo.co.in

Department of Bacteriology, National Institute for Research in Tuberculosis (Indian Council of Medical Research), Chennai, India
}

processed sputum samples for the rapid recovery of Mycobacterium tuberculosis [5]. The phages as such were unable to control some strains of Bacillus $s p$ and Staphylococcus $s p$. Moreover, they were temperate in nature leading to the growth of lysogens after 24 hours of phage addition and incubation. To circumvent this situation, phage lysins extracted from all the three phages were pooled and used to supplement phagebiotics for the effective control of normal flora and to prevent the formation of lysogens in processed sputum samples [6]. When phages supplemented with lysins, normal flora were effectively controlled but the lysogens were formed after 24 hours of addition as the lysins were used along with phages. However, the use of phage lysins alone was found to control normal flora as well as to prevent the risk of lysogen development [7].

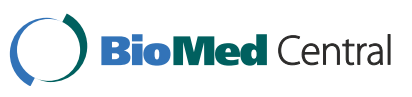


Pooled phage lysin was evaluated earlier in comparison with antibiotics to control the growth of normal flora in processed sputum samples for the detection of $M$. tuberculosis using BACTEC MGIT 960 system. The results indicated that phage lysin was comparable to antibiotic supplement in decontaminating sputum samples and in detecting $M$. tuberculosis [8]. The present work was aimed at evaluating phage lysin in the rapid detection of M. tuberculosis directly from the processed sputum samples using luciferase reporter phage (LRP) assay.

\section{Methods}

\section{Luciferase reporter phage}

Luciferase reporter phage, phAE129 was used for this study to detect $M$. tuberculosis from sputum specimens. The phage was propagated to get a high titer and maintained at $4^{\circ} \mathrm{C}$. A titre of $1 \times 10^{-9}$ was used throughout the study.

\section{Phage lysin}

Three bacteriophages namely Chedec 11, Chedec 20 and Chedec 21 were used for the lysin extraction as these phages showed broad host range of infecting more normal flora present in the processed sputum samples [5]. Phage lysin was prepared individually from phages Chedec 11, Chedec 20 and Chedec 21 as described earlier [6]. The clear supernatant fluid with its active lysin obtained during the final stage of lysin preparation was frozen at $-20^{\circ} \mathrm{C}$ [6]. The final precipitate was protein estimated and found to be $0.158,0.138$ and $0.125 \mathrm{mg} / \mathrm{ml}$ respectively for Chedec 11, Chedec 20 and Chedec 21. These phage lysins were pooled prior to use. The working concentration of phage lysin was prepared by mixing $0.8 \mathrm{ml}$ of pooled phage lysins with $7 \mathrm{ml}$ of $7 \mathrm{H} 9$ medium supplemented with $0.5 \%$ glycerol and 10\% albumin dextrose complex (D-glucose-4 grams; $\mathrm{NaCl}-0.85$ gram; Bovine serum albumin-10 grams in $100 \mathrm{ml}$ of distilled water).

\section{Diagnostic luciferase reporter phage assay (DLRPA) for the detection of M. Tuberculosis}

Sputum specimens used in this study were obtained from patients enrolled in controlled clinical trials of the National Institute for Research in Tuberculosis (NIRT), Chennai. All the clinical trials conducted at NIRT were approved by institutional ethical committee members and due informed consent was also obtained from patients. A total of 129 sputum specimens were collected; one smear was prepared from each sample and stained by auramine phenol staining method [9]. The specimens were processed by modified Petroff's method and the deposits thus obtained were washed with $0.067 \mathrm{M}$ phosphate buffer saline at $\mathrm{pH} 7.0$ to neutralize the samples. Two Lowenstein Jensen (LJ) slopes were inoculated from the deposits (Petroff's LJ culture). The LJ slopes were randomized, incubated at $37^{\circ} \mathrm{C}$ and read every week up to 8 weeks. The remaining deposits were transferred to $7 \mathrm{ml}$ of $7 \mathrm{H} 9$ complete medium containing phage lysin and randomized. The samples were incubated at $37^{\circ} \mathrm{C}$ as a standing culture without shaking. At the end of day 7, 9, 14 and 21, about $500 \mu \mathrm{l}$ of the samples were withdrawn and mixed with $40 \mu \mathrm{l}$ of $0.1 \mathrm{M}$ $\mathrm{CaCl}_{2}$ and $100 \mu \mathrm{l}$ of phAE129 and incubated at $37^{\circ} \mathrm{C}$ for 4-6 hours. After incubation, $100 \mu \mathrm{l}$ of the sample was mixed with $100 \mu \mathrm{l}$ of $0.33 \mathrm{M}$ D-luciferin dissolved in $0.05 \mathrm{M}$ sodium citrate buffer at $\mathrm{pH} 4.5$. Relative light unit (RLU) was measured in a luminometer (Monolight 2010) at 10 seconds integration time. RLU was measured in duplicates for all the samples and mean value was used for interpretation of results. A drop of each sample was placed on sheep blood agar plate to check for contamination at every time point. At the end of day 21, all the samples were centrifuged at 3500 RPM for 15 minutes and the pellets were inoculated on to $2 \mathrm{LJ}$ slopes to check whether LRP positives are true positives. The LJ slopes were randomized, incubated at $37^{\circ} \mathrm{C}$ and read every week up to 8 weeks (Final LJ culture). The results were compared after decoding. The samples resulted as contamination in either of the methods were excluded from the comparison.

\section{Statistical analysis}

The test of significance was calculated by Pearson Chi-Square test using SPSS software version 14.

\section{Results}

Among 129 sputum specimens, 95 samples were positive (74\%) and 34 samples were negative (26\%) for M. tuberculosis by smear using auramine phenol staining method. Likewise, 48 samples were negative (37.2\%) and 70 samples were positive $(54.3 \%)$ by LJ culture and 10 samples resulted in the growth of unclassified mycobacteria $(7.8 \%)$ while 1 sample was contaminated $(0.7 \%)$. The sensitivity and specificity of DLRPA for detecting $M$. tuberculosis from sputum specimens was $90 \%$ and $81 \%$ respectively compared to conventional culture. The agreement between the methods was $87 \%$ (Table 1). Either the samples resulted as non tuberculous mycobacteria (NTM) or contamination were excluded from the comparison.

\begin{tabular}{|c|c|c|c|c|}
\hline & \multicolumn{4}{|c|}{ Petroff's culture } \\
\hline & Positive & $\begin{array}{c}\text { Positive } \\
63\end{array}$ & $\begin{array}{c}\text { Negative } \\
8\end{array}$ & $\begin{array}{c}\text { Total } \\
71\end{array}$ \\
\hline \multirow[t]{2}{*}{ DLRPA } & Negative & 7 & 34 & 41 \\
\hline & Total & 70 & 42 & 112 \\
\hline
\end{tabular}

Sensitivity $90 \%$; Specificity $81 \%$; Agreement $87 \%$. 
Among 48 culture negative samples, 8 samples were positive $(16.6 \%)$, and 6 samples were contaminated by DLRPA. DLRPA was unable to detect two $1+$, two $2+$ and three few-colony-growth of culture positive samples (7 out of 70). Among 10 samples that resulted in the growth of NTM, 5 samples were negative and another 5 samples were contaminated by DLRPA. One sample contaminated by conventional LJ culture was also contaminated by DLRPA. The average time to detection for detecting culture negatives, few-colony-growth, $1+$ (20-100 colonies), $2+$ (>100 colonies) and $3+$ (confluent growth) by DLRPA was 19, 19, $15.45,10.88$ and 8.14 days respectively with a median TTD of 14.5 days (Table 2). The earliest time points at which $M$. tuberculosis was detected by DLRPA for each culture grade are given in Table 2 .

DLRPA was found to detect most of the $3+$ samples at day 7 while $2+$ samples were found to be detected at day 7 and 9. Most of the 1+, few-colony-growth and negatives samples were detected as positive by DLRPA at day 21 . Eighty-nine percent (41out of 46) of the higher grade culture positive samples $(3+$ and $2+)$ were positive by DLRPA at more than one time points of RLU readings between day 7 and day 21 while $60 \%$ (15 out of 25 ) of the lower grade $(1+$, few-colony-growth) and culture negative samples were positive only at the end of day 21 . The rate of contamination for DLRPA using phage lysin was 9.3\%.

The "final LJ culture" confirmed the positives by DLRPA in 64 out of 71 samples. Of the remaining 7 samples, 6 samples were negative and one was contaminated by "final LJ culture". DLRPA failed to detect 4 samples (two 1+ samples, one $3+$ sample and one 1 colony sample) that resulted in growth in "final LJ culture".

\section{Statistical analysis}

DLRPA was found to be statistically significant compared to conventional culture for the detection of M. tuberculosis directly from the processed sputum specimens. The Pearson Chi-Square test showed a value of 0.000 . The $\mathrm{p}$ value was 0.000001 .

\section{Discussion}

Rapid detection, identification and drug susceptibility testing of $M$. tuberculosis is important for better patient management. Delay in diagnosis and treatment is an important contributor for rapid spread of TB in the community [10] leading to excessive morbidity and mortality especially in HIV infected patients [11]. This delay is also responsible for increased nosocomial outbreaks among patients and health care workers [12].

Growing $M$. tuberculosis in liquid medium is much faster when compared to conventional method of detection using solid LJ medium. The major limitation in using liquid medium for the primary isolation of $M$. tuberculosis is the over growth of normal flora which compete for nutrients and mask the growth of tubercle bacilli. Antibiotics are in use either in form of PANTA or PACT to control the growth of normal flora. But it is recommended that exposure of primary isolates to these selective antibiotics should be limited as these agents affect the growth of mycobacteria in liquid media. As an alternative to antibiotics, bacteriophages and their lysins were used in liquid medium to control the growth of normal flora for the detection of $M$. tuberculosis.

Use of liquid culture systems such as BACTEC MGIT 960 has significantly reduced the time for detection and drug susceptibility testing of mycobacteria [13]. The higher initial investment for the equipment and further recurring charges for the procurement of MGIT vials is a major limitation for MGIT 960 system especially in resource limited settings [14]. Phage lysin was evaluated initially in comparison with PANTA to control the overgrowth of normal of normal flora for the recovery of $M$. tuberculosis using BACTEC MGIT 960 system. Phage lysin was found to be comparable to PANTA to control the growth of normal flora.

Mycobacteriophage based methods have been developed for the rapid detection and drug sensitivity testing of $M$. tuberculosis. Among phage based assays, luciferase reporter phage assay has the potential for the detection,

Table 2 DLRPA for the detection of $M$. tuberculosis from sputum samples

\begin{tabular}{|c|c|c|c|c|c|c|c|c|c|c|}
\hline \multirow{2}{*}{$\begin{array}{l}\text { Culture } \\
\text { grade } \\
\text { on LJ }\end{array}$} & \multirow{2}{*}{$\begin{array}{c}\text { No. of } \\
\text { samples }\end{array}$} & \multicolumn{3}{|c|}{ LRP } & \multicolumn{4}{|c|}{ Early time of detection (days) ${ }^{@}$} & \multicolumn{2}{|c|}{ Average TTD (in days) } \\
\hline & & Pos (\%) & Neg & Cont & 7 & 9 & 14 & 21 & LRP & LJ \\
\hline Negatives & 48 & 8 (16.6) & 34 & 6 & 0 & 1 & 1 & 6 & 19 & NA \\
\hline Colonies & 9 & $6(66.6)$ & 3 & 0 & 1 & 0 & 0 & 5 & 19 & 38.5 \\
\hline $1+$ & 13 & $11(84.6)$ & 2 & 0 & 3 & 1 & 1 & 6 & 15.45 & 29.27 \\
\hline $2+$ & 26 & $24(92.3)$ & 2 & 0 & 8 & 8 & 5 & 3 & 10.88 & 20.41 \\
\hline $3+$ & 22 & $22(100)$ & 0 & 0 & 17 & 2 & 3 & 0 & 8.14 & 14 \\
\hline NTM & 10 & 0 & 5 & 5 & 0 & 0 & 0 & 0 & NA & 23.1 \\
\hline Cont & 1 & 0 & 0 & 1 & 0 & 0 & 0 & 0 & NA & NA \\
\hline
\end{tabular}

Pos: Positives; Neg: Negatives; Cont: Contaminated; NTM: Non-tuberculous mycobacteria; TTD: Time-to-detection; NA: Not applicable; LJ: Petroff's culture; Colonies: 1-19 colonies on LJ; 1+: 20-100 colonies; $2+$ : >100 colonies; $3+$ : confluent growth of M. tuberculosis.

@ Number of samples detected at early time (i.e. between 7 to 21 days) by D. 
identification and drug sensitivity testing of M. tuberculosis [15]. LRP assay is utilized to study the drug susceptibility profile of tubercle bacilli using primary culture obtained from LJ medium. The presence of mucus strands, normal flora, enzymes and inhibitory factors in sputum specimens forms the major hurdle in developing a standard diagnostic assay.

Since LRP assay involves frequent opening of the culture vials for taking out aliquots at different days for setting up the assay, it requires stringent measures to prevent the overgrowth of non mycobacterial contaminants especially from environmental organisms. Combination of antibiotics (PANTA) was used in most of the liquid culture detection systems available for mycobacteria to decontaminate processed sputum samples. PANTA was also used to control the overgrowth of normal flora in sputum samples processed for M. tuberculosis detection by LRP assay $[15,16]$. Earlier, lysin was evaluated in comparison with PANTA to control the overgrowth of normal flora normal in processed sputum samples for detection of $M$. tuberculosis by BACTEC MGIT 960 system [8]. Here we reported a simple and bio-friendly methodology to control surviving normal flora using phage lysin in place of antibiotics for luciferase reporter phage assay with improved sensitivity and specificity for the detection of $M$. tuberculosis.

Luciferase reporter phage assay had been recognized for the rapid assessment of drug susceptibility of Mycobacterium tuberculosis. The time required for the identification of antibiotics sensitivity pattern of $M$. tuberculosis was reduced from weeks to days using LRP assay [17]. Being a viability based test, LRP was also utilized for the rapid screening of new antituberculosis drugs [18,19]. Mycobacteriophages specific for $M$. tuberculosis alone were used for simultaneous identification and drug susceptibility testing of $M$. tuberculosis cultures grown from MGIT 960 system. Luciferase reporter mycobacteriophage phAE142 was utilized for this purpose [20]. Based on the rapidity, specificity and the ability of LRP assay to detect viable tubercle bacilli, the present work is aimed to improve upon the sensitivity of detection using phage lysin and avoiding the use of antibiotics.

In 2003, Bardarov et al., [16] reported that LRP can be used for the rapid detection of $M$. tuberculosis in conventionally processed sputum samples but the assay required a minimum of $>10^{7} \mathrm{CFU} / \mathrm{ml}$ as a starting inoculum. Allowing a brief period of time to grow $M$. tuberculosis in liquid media, light can be detected at a rate faster than the doubling time of organisms was also suggested. Accordingly, the samples were incubated at $37^{\circ} \mathrm{C}$ and tested by LRP infection at each days starting from 1 to 42 to improve the sensitivity of detection. The present approach is also corroborating the earlier methodology but LRP infection by phAE129 is restricted to days 7, 9, 14 and 21 for the detection of $M$. tuberculosis. The results also suggest that the samples containing higher CFU can be detected in $\leq 7$ days and the samples with low CFU can be detected between 7 to 21 days. Incubation of samples for more than 21 days probably would result in increased sensitivity of $M$. tuberculosis detection especially from paucibacillary samples.

Phage phAE142 was used for the direct detection of $M$. tuberculosis from processed sputum samples [15]. The results were compared with MGIT 960 and conventional culture on LJ. LRP infection was done on post incubation days $1,3,5,7,11,15,19,23,27,41$ and 55. LRP detected M. tuberculosis in a range between 1 and 41 days with a mean time to detection of 7 days. The sensitivity of LRP assay was $76 \%$ while $97 \%$ and $90 \%$ of the samples were positive by MGIT 960 and LJ respectively. The sensitivity of detection by LRP was improved from $76 \%$ to $92 \%$ when contaminated specimens were excluded. In the present work, though the median TTD is 14.5 days, the sensitivity of detecting M. tuberculosis is as high as $90 \%$ with phAE129 using a simple methodology.

In the present work, 12 out of 129 samples were contaminated by DLRPA when the growth of other mycobacteria is included in the comparison. Among these 12 samples, 5 samples were reported as non-tuberculous mycobacteria (NTM) by conventional LJ method. In principle, all the NTMs also will be considered as contamination by conventional LJ. But because of the limitation of DLRPA to confirm all these NTMs, the samples were reported as contamination. If the NTMs were excluded from the comparison, the rate of contamination for DLPRA would be only $5.4 \%$. Similarly, if consider the total mycobacteria isolated the positivity rate for LJ was $62 \%$ (80 out of 129 ) but for DLRPA it was only 55\% (71/129). If the NTMs were excluded, the positivity rate for LJ was $54 \%$ and for DLRPA it remains $55 \%$.

Luciferase reporter phage assay has certain advantages over the other liquid based rapid diagnostic assays while detecting $M$. tuberculosis directly from processed sputum samples. The methodology of this rapid assay is very simple and can be easily adopted by any laboratory. The average TTD of "BACTEC MGIT 960", the current Gold-standard for liquid medium, has been reported to range between 8.5 [21] and 13.3 [22] days. The median TTD of DLRPA is 14.5 as observed in the present study. Among the 10 nontuberculous mycobacteria grown on LJ, 5 resulted in contamination while growing on liquid medium; the other 5 became negative by DLPRA which reiterates that the phage used in this study is highly specific in infecting $M$. tuberculosis. As only viable bacterial cell will support phage adsorption, infection, replication and subsequent progeny release, the assay detects the active disease. As an indigenous method, propagation, harvest and maintenance of phages are relatively simple and less laborious. The costeffectiveness of the assay is highly dependent on the cost of luminometer and the substrate, D-luciferin. 


\section{Conclusion}

DLRPA, done with phage lysin, is a simple, rapid, highly specific, viability based, bio-friendly, in-house methodology that has the potential to be developed as a point of care system for the rapid detection and drug sensitivity testing of $M$. tuberculosis. DLRPA can be implemented for the rapid availability of diagnostic results for $M$. tuberculosis especially in countries endemic to tuberculosis and in resource limited settings.

\section{Abbreviations}

LRP: Luciferase Reporter phage; DLRPA: Diagnostic Luciferase Reporter Phage Assay; TTD: Time To Detection; CFU: Colony Forming Unit; PANTA: Polymixin B, Amphotericin B, Nalidixic acid, Trimethoprim, Azlocillin; PACT: Polymixin B, Amphotericin B, Carbenicillin, Trimethoprim; MGIT: Mycobacterial Growth Indicator Tube.

\section{Competing interests}

The authors declare that they have no competing interest.

\section{Authors' contributions}

BS was responsible for the laboratory work and writing of the manuscript. GS was for responsible for the interpretation of the results and data analysis. AD was responsible for the data interpretation. VK was responsible for the study design and overall supervision. All authors read and approved the final manuscript.

\section{Acknowledgement}

Balaji Subramanyam acknowledges the financial support from Indian Council of Medical Research, New Delhi, INDIA. Authors acknowledge the statistical support rendered by Dr. C. Ponnuraja, Scientist 'B', Department of Statistics, National Institute for Research in Tuberculosis.

Received: 28 June 2012 Accepted: 17 January 2013

Published: 28 January 2013

\section{References}

1. Rosovitz MJ, Leppla SH: Virus deals anthrax a killer blow. Nature 2002, 418:825-826.

2. Borysowski J, Weber-Dabrowska B, Gorski A: Bacteriophage endolysins as a novel class of antibacterial agents. Exp Biol Med (Maywood) 2006, 231:366-377.

3. Fischetti VA: Bacteriophage lysins as effective antibacterials. Curr Opin Microbiol 2008, 11:393-400.

4. O'Flaherty S, Ross RP, Coffey A: Bacteriophage and their lysins for elimination of infectious bacteria. FEMS Microbiol Rev 2009, 33:801-819.

5. Kumar V, Balaji S, Gomathi NS, Venkataesan P, Gomathi S, Jayasankar K, Narayanan PR: Phage cocktail to control the exponential growth of normal flora in processed sputum specimens grown overnight in liquid medium for rapid TB diagnosis. J Microbiol Methods 2007, 68:536-542.

6. Subramanyam B, Kumar V, Perumal V, Nagamiah S: Phage lysin to supplement phagebiotics to decontaminate processed sputum specimens. Eur J Clin Microbiol Infect Dis 2010, 29:1407-1412.

7. Subramanyam B, Kumar V: Effect of bacteriophage lysin on lysogens. Asian Pacific J Tropical Med 2011, 4:306-308.

8. Subramanyam B, Sivaramakrishnan GN, Dusthackeer A, Nagamiah S, Kumar $\checkmark$ : Phage lysin as a substitute for antibiotics to detect Mycobacterium tuberculosis from sputum samples with the BACTEC MGIT 960 system. Clin Microbiol Infect 2011, 18(5):497-501.

9. Selvakumar N, Sudhamathi S, Duraipandian M, Frieden TR, Narayanan PR: Reduced detection by Ziehl-Neelsen method of acid-fast bacilli in sputum samples preserved in cetylpyridinium chloride solution. Int J Tuberc Lung Dis 2004, 8:248-252.

10. Basnet R, Hinderaker SG, Enarson D, Malla P, Morkve O: Delay in the diagnosis of tuberculosis in Nepal. BMC Public Health 2009, 9:236.

11. Meintjes G, Schoeman H, Morroni C, Wilson D, Maartens G: Patient and provider delay in tuberculosis suspects from communities with a high HIV prevalence in South Africa: a cross-sectional study. BMC Infect Dis 2008, 8:72.
12. Greenaway C, Menzies D, Fanning A, Grewal R, Yuan L, FitzGerald JM: Delay in diagnosis among hospitalized patients with active tuberculosispredictors and outcomes. Am J Respir Crit Care Med 2002, 165:927-933.

13. Hanna BA, Ebrahimzadeh A, Elliott LB: Multicenter evaluation of the BACTEC MGIT 960 system for recovery of mycobacteria. J Clin Microbiol 1999, 37:748-752.

14. Rodrigues C, Shenai S, Sadani M: Evaluation of the bactec MGIT 960 TB system for recovery and identification of Mycobacterium tuberculosis complex in a high through put tertiary care centre. Indian J Med Microbiol 2009, 27:217-221.

15. Banaiee N, Bobadilla-Del-Valle M, Bardarov S: Luciferase reporter mycobacteriophages for detection, identification, and antibiotic susceptibility testing of Mycobacterium tuberculosis in Mexico. J Clin Microbiol 2001, 39:3883-3888.

16. Bardarov S Jr, Dou H, Eisenach K: Detection and drug-susceptibility testing of $M$. tuberculosis from sputum samples using luciferase reporter phage: comparison with the Mycobacteria Growth Indicator Tube (MGIT) system. Diagn Microbiol Infect Dis 2003, 45:53-61.

17. Jacobs WR Jr, Barletta RG, Udani R: Rapid assessment of drug susceptibilities of Mycobacterium tuberculosis by means of luciferase reporter phages. Science 1993, 260:819-822.

18. Raparti V, Chitre T, Bothara K: Novel 4-(morpholin-4-yl)-N'-(arylidene) benzohydrazides: synthesis, antimycobacterial activity and QSAR investigations. Eur J Med Chem 2009, 44:3954-3960.

19. Sivakumar PM, Prabhu Seenivasan S, Kumar V, Doble M: Novel 1,3,5triphenyl-2-pyrazolines as anti-infective agents. Bioorg Med Chem Lett 2010, 20:3169-3172.

20. Banaiee N, Bobadilla-del-Valle M, Riska PF: Rapid identification and susceptibility testing of Mycobacterium tuberculosis from MGIT cultures with luciferase reporter mycobacteriophages. J Med Microbiol 2003, 52:557-561.

21. Whyte T, Hanahoe B, Collins T, Corbett-Feeney G, Cormican M: Evaluation of the BACTEC MGIT 960 and MB BAC/T systems for routine detection of Mycobacterium tuberculosis. J Clin Microbiol 2000, 38:3131-3132.

22. Tortoli E, Cichero P, Piersimoni C, Simonetti MT, Gesu G, Nista D: Use of BACTEC MGIT 960 for recovery of mycobacteria from clinical specimens: multicenter study. J Clin Microbiol 1999, 37:3578-3582.

doi:10.1186/1471-2334-13-44

Cite this article as: Subramanyam et al:: Phage lysin to control the overgrowth of normal flora in processed sputum samples for the rapid and sensitive detection of Mycobacterium tuberculosis by luciferase reporter phage assay. BMC Infectious Diseases 2013 13:44.

\section{Submit your next manuscript to BioMed Central and take full advantage of:}

- Convenient online submission

- Thorough peer review

- No space constraints or color figure charges

- Immediate publication on acceptance

- Inclusion in PubMed, CAS, Scopus and Google Scholar

- Research which is freely available for redistribution 